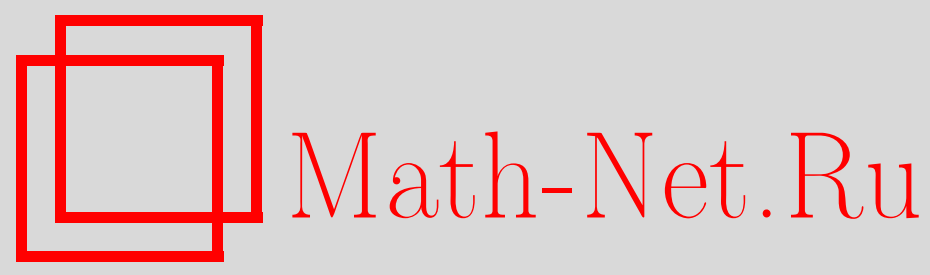

П. А. Терехин, Базисы Рисса, порожденные сжатиями и сдвигами функции на отрезке, Матем. заметки, 2002, том 72 , выпуск 4, 547-560

DOI: https://doi.org/10.4213/mzm444

Использование Общероссийского математического портала Math-Net.Ru подразумевает, что вы прочитали и согласны с пользовательским соглашением http://www . mathnet.ru/rus/agreement

Параметры загрузки:

IP: 34.227 .88 .159

26 апреля 2023 г., $16: 36: 22$

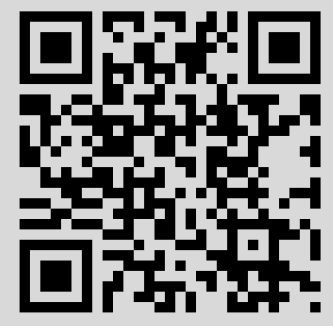


УДК 517.51

\section{БАЗИСЫ РИССА, ПОРОЖДЕННЫЕ СЖАТИЯМИ И СДВИГАМИ ФУНКЦИИ НА ОТРЕЗКЕ}

\section{П. А. Терехин}

Даны условия базисности по Риссу систем сжатий и сдвигов функции.

Библиография: 6 названий.

\section{1. Формулировки теорем}

Пусть функция $\varphi(x)$ задана на полуинтервале $[0,1)$ и принимает, вообще говоря, комплексные значения.

Для $n \in \mathbb{N}$ по стандартному представлению $n=2^{k}+j, k \in \mathbb{P}=\mathbb{N} \cup\{0\}, 0 \leqslant j<2^{k}$, обозначая $\Delta_{i}^{(k)}=\left[j / 2^{k},(j+1) / 2^{k}\right)$, полагаем

$$
\varphi_{n}(x)=\varphi_{k j}(x)= \begin{cases}2^{k / 2} \varphi\left(2^{k} x-j\right), & x \in \Delta_{j}^{(k)}, \\ 0, & x \notin \Delta_{j}^{(k)} .\end{cases}
$$

Пусть, кроме того, $\varphi_{0}=\chi[0,1)-$ характеристическая функция $[0,1)$.

ОПРЕДЕЛЕНИЕ 1. Семейство $\left(\varphi_{n}\right), n \in \mathbb{P}$, называем системой сдвигов и сжатий функиии $\varphi$.

Классическая система Хаaра $\left(\chi_{n}\right)$, где $\chi_{0}=\chi[0,1)$ и

$$
\chi_{n}(x)=\chi_{k j}(x)= \begin{cases}2^{k / 2}, & x \in \Delta_{2 j}^{(k+1)}, \\ -2^{k / 2}, & x \in \Delta_{2 j+1}^{(k+1)}, \\ 0, & x \notin \Delta_{j}^{(k)},\end{cases}
$$

для $n=2^{k}+j$ (стандартное представление), является, как нетрудно видеть, системой сжатий и сдвигов функции $\chi=\chi[0,1 / 2)-\chi[1 / 2,1)$.

В статье изучается

Вопрос. При каких условиях на функцию $\varphi$ система ее сжатий и сдвигов есть базис пространства $L_{2}[0,1]$, эквивалентный ортонормированному (базис Рисса), т.е. $\varphi_{n}=$ $A e_{n}$, где $A$ - обратимый и вместе с $A^{-1}$ ограниченный оператор в $L_{2}[0,1],\left(e_{n}\right)$ - ортонормированньй базис.

Отметим, что вообще для базисности системы сжатий и сдвигов функции $\varphi$ принципиально необходимо, чтобы $\int_{0}^{1} \varphi(x) d x=0$. В ином случае, как следует из результатов Филиппова и Освальда [1], система переполнена и потому базисом быть не может. 
В статье указьваются два способа построения базисов Рисса. Первый из них заключается в следующем.

Пусть $H^{\infty}$ - банахова алгебра аналитических в открытом единичном круге функций, $G\left(H^{\infty}\right)$ - группа обратимых элементов алгебры $H^{\infty}$. Заметим, что для принадлежности $u \in G\left(H^{\infty}\right)$ необходимо и достаточно, чтобы функция $u(z)$ была аналитической в круге $|z|<1$ и чтобы в этом круге вьполнялись неравенства

$$
0<\inf |u(z)|, \quad \sup |u(z)|<\infty \text {. }
$$

Теорема 1. Пусть система $\left(\psi_{n}\right)$ сжатий и сдвигов функиии $\psi$ является базисом Рисса, и пусть разложсение функции ч по системе $\left(\psi_{n}\right)$ имеет вид

$$
\varphi=\sum_{k=0}^{\infty} 2^{-k / 2} c_{k} \sum_{j=0}^{2^{k}-1} \psi_{k j}
$$

Тогда для того чтобы система $\left(\varphi_{n}\right)$ сжстай и сдвигов функиии ч была базисом Рисса, необходимо и достаточно, чтобы функиия

$$
u(z)=\sum_{k=0}^{\infty} c_{k} z^{k}
$$

принадлежсала әруппе $G\left(H^{\infty}\right)$.

ПРИмЕчАнИЕ 1. В качестве функции $\psi$ в посылке теоремы можно взять, например, функцию $\psi$, порождающую систему Хаара.

ПРИМЕЧАНИЕ 2. ФункциИ

$$
2^{-k / 2} \sum_{j=0}^{2^{k}-1} \psi_{k j}
$$

фигурирующие в разложении (1.3), являются формальными аналогами функций Радемахера, соответствующих случаю $\psi=\chi$.

Второй способ построения базисов Рисса дается теоремой 2 , основанной на следующем определении.

ОПрЕДЕЛЕниЕ 2. Пусть $\left(b_{k}\right), k \in \mathbb{N},-$ последовательность неотрищательных чисел, суммируемая с квадратом: $b_{k} \geqslant 0, \sum b_{k}^{2}<\infty$; функция $\psi$ такова, что система ее сжатий и сдвигов является базисом Рисса. Обозначим через $A\left(\psi,\left(b_{k}\right)\right)$ - класс функиий $\varphi$, разлохсение которых по $\left(\psi_{n}\right)$

$$
\varphi=c_{0} \psi+\sum_{k=1}^{\infty} \sum_{j=0}^{2^{k}-1} c_{k j} \psi_{k j}
$$

удовлетворяет условиям

$$
c_{0} \neq 0, \quad \sum_{j=0}^{2^{k}-1}\left|c_{k_{j}}\right|^{2} \leqslant\left(\left|c_{0}\right| b_{k}\right)^{2}, \quad k \in \mathbb{N} .
$$


ТЕОРема 2. Для того чтобы для любой функиии $\varphi \in A\left(\psi,\left(b_{k}\right)\right)$ система ее сжатий и сдвигов была базисом Рисса, необходимо и достаточно, чтобы выполнялось неравенство

$$
\sum_{k=1}^{\infty} b_{k}<1
$$

ПримечаниЕ 3 . Классы $A\left(\psi,\left(b_{k}\right)\right)$ являются аналогами классов $A_{\alpha}(g, M)$, определенных Сабуровой $[2]$ в связи с вопросом базисности в $C[0,1]$.

ПРИМЕЧАНИЕ 4. Поскольку свойство базисности по Риссу однородно (не зависит от умножения $\varphi$ на ненулевой числовой множитель), то в (1.4) и (1.5) можно считать $c_{0}=1$.

\section{2. Вспомогательные утверждения}

ОБознАчения. Пусть $L_{2}^{0}$ - пространство функций, определенных на $[0,1)$, интегрируемых с квадратом и имеющих нулевой интеграл;

$r_{k}=2^{-k / 2} \sum_{j=0}^{2^{k}-1} \chi_{k j}-$ функции Радемахера, $k \in \mathbb{P}$ :

$k(n), j(n)$ определяются из стандартного представления (см. раздел 1$)$ натурального $n=2^{k}+j$ по правилу $k(n)=k, j(n)=j$;

$\prod_{\nu=1}^{k} A_{\nu}$ - произведение операторов $A_{\nu}$ в следующем порядке: $A_{1} \cdots A_{k}$ (первым действует оператор $A_{k}$, последним - $A_{1}$ ), при $k=0$ пустое произведение считаем равным тождественному оператору.

Здесь и далее под словом оператор понимаем ограниченньй линейньй оператор, действуюший в пространстве $L_{2}^{0}$.

2.1. Предложения. Определим операторы сжатия-сдвига $S_{n}, n \in \mathbb{N}$, посредством равенства

$$
S_{n} \varphi=\varphi_{n},
$$

где $\varphi_{n}$ - элемент системы сжатий и сдвигов функции $\varphi$ (см. раздел 1$)$.

Корректность определения (линейность и ограниченность сжатия-сдвига) нетрудно проверить, пользуясь равенством (1.1).

ПРЕДЛОЖЕНИЕ 1. Справедливо представление

$$
S_{n}=\prod_{\nu=1}^{k} S\left(\varepsilon_{\nu}\right)
$$

где $k=k(n), \varepsilon_{\nu}$ определяются из двоичного разложения $j(n)=\sum_{\nu=1}^{k} \varepsilon_{\nu} 2^{k-\nu}$ так, что $\varepsilon_{\nu}=0$ или 1 ; операторы $S(\varepsilon), \varepsilon \in\{0,1\}$, определяются из равенства

$$
S(\varepsilon) \varphi(x)= \begin{cases}2^{1 / 2} \varphi(2 x-\varepsilon), & x \in[\varepsilon / 2,(\varepsilon+1) / 2), \\ 0, & x \notin[\varepsilon / 2,(\varepsilon+1) / 2) .\end{cases}
$$


ДокАЗАТЕЛЬСТво. Продолжим функцию $\varphi \in L_{2}^{0}$ нулем за полуинтервал $[0,1)$. Будем иметь

$$
\begin{aligned}
S_{n} \varphi(x) & =\varphi_{n}(x)=\varphi_{k j}(x)=2^{k / 2} \varphi\left(2^{k} x-j\right) \\
& =2^{k / 2} \varphi\left(2^{k} x-2^{k-1} \varepsilon_{1}-\cdots-2 \varepsilon_{k-1}-\varepsilon_{k}\right) \\
& =2^{1 / 2} \cdots 2^{1 / 2} \varphi\left(2\left(2 \cdots\left(2 x-\varepsilon_{1}\right)-\cdots-\varepsilon_{k-1}\right)-\varepsilon_{k}\right)=S\left(\varepsilon_{1}\right) \cdots S\left(\varepsilon_{k}\right) \varphi(x) .
\end{aligned}
$$

Учли (2.1), определение 1 функций $\varphi_{n}$ и пояснения к (2.2).

ПРЕДЛОЖЕНИЕ 2. Для оператора $А$ следующие условия әквивалентны:

(а) для некоторой функиии $\psi$ имеем $A \chi_{n}=\psi_{n}, n \in \mathbb{N}$;

(б) А перестановочен с $S_{n}, n \in \mathbb{N}$;

(в) если $A \omega=\varphi$, то $A \omega_{n}=\varphi_{n}$ для всех $n \in \mathbb{N}$.

ДокАЗАТЕЛЬСтво. Из (а) следует (б). В самом деле, условие (а) ввиду определения (2.1) оператора $S_{n}$ можно записать в виде $A S_{n} \chi=S_{n} A \chi$.

Пусть $p, q \in \mathbb{N}$. Согласно представлению (2.2) произведение $S_{p} S_{q}$ равно $S_{n}$ для некоторого $n \in \mathbb{N}$. Отсюда, учитьвая дважды равенство (2.1) для $q$ и последнее равенство (для $n$ и для $q$ ), находим

$$
A S_{p} \chi_{q}=A S_{p} S_{q} \chi=A S_{n} \chi=S_{n} A \chi=S_{p} S_{q} A \chi=S_{p} A S_{q} \chi=S_{p} A \chi_{q} .
$$

В силу полноты в пространстве $L_{2}^{0}$ системы $\left(\chi_{q}\right), q \in \mathbb{N}$, получаем $A S_{p} f=S_{p} A f$ для любой функции $f \in L_{2}^{0}$, что означает условие (б).

Из (б) следует (в). В самом деле, согласно определению (2.1) оператора $S_{n}$ и посылке условия (в) имеем $A \omega_{n}=A S_{n} \omega=S_{n} A \omega=S_{n} \varphi=\varphi_{n}$.

Из (в) следует (а). Достаточно положить $\psi=A \chi$.

ПРЕДЛОЖЕНИЕ 3. Для степеней оператора $U=2^{-1 / 2}(S(0)+S(1))$ имеем

$$
U^{k} \varphi=2^{-k / 2} \sum_{j=0}^{2^{k}-1} \varphi_{k j}
$$

в частности,

$$
U^{k} \chi=r_{k}
$$

ДокАзАТЕЛЬСТво. Имея в виду определение оператора $U$, находим

$$
U^{k}=2^{-k / 2}(S(0)+S(1))^{k}=2^{-k / 2} \sum_{\varepsilon_{1}, \ldots, \varepsilon_{k}} \prod_{\nu=1}^{k} S\left(\varepsilon_{\nu}\right)=2^{-k / 2} \sum_{k(n)=k} S_{n}
$$

- в последнем равенстве учли представление (2.2).

Тогда

$$
U^{k} \varphi=2^{-k / 2} \sum_{k(n)=k} S_{n} \varphi=2^{-k / 2} \sum_{k(n)=k} \varphi_{n}=2^{-k / 2} \sum_{j=0}^{2^{k}-1} \varphi_{k j}
$$

мы учли (2.1) и определение системы сжатий и сдвигов из раздела 1. Равенство (2.3) установлено. Полагая в нем $\varphi=\chi$, получаем (2.4) (см. обозначение $r_{k}$ ). 
ПреДЛОЖЕнИЕ 4. Пусть $k, m \in \mathbb{P}, 0 \leqslant j<2^{k}, 0 \leqslant i<2^{m}, u$ функция $\varphi$ представима рядом по системе Радемахера

$$
\varphi=\sum_{\nu=0}^{\infty} c_{\nu} r_{\nu}
$$

\section{Тогда выполняются равенства}

$$
\begin{aligned}
\sum_{j=0}^{2^{k}-1}\left(\varphi_{k j}, \chi_{m i}\right) & =2^{-(m-k) / 2} c_{m-k}, & & \text { если } k<m ; \\
\left(\varphi_{k j}, \chi_{m i}\right) & =\delta_{i j} c_{0}, & & \text { если } k=m ; \\
\left(\varphi_{k j}, \chi_{m i}\right) & =0, & & \text { если } k>m .
\end{aligned}
$$

ДокАЗАТЕЛЬСтво. Пусть сначала $k<m$.

Согласно посылке предложения и равенству (2.4) имеем

$$
U^{k} \varphi=U^{k} \sum_{\nu=0}^{\infty} c_{\nu} r_{\nu}=\sum_{\nu=0}^{\infty} c_{\nu} U^{k+\nu} \chi=\sum_{\nu=0}^{\infty} c_{\nu} r_{k+\nu}
$$

откуда, используя $(2.3)$, получаем

$$
\sum_{j=0}^{2^{k}-1}\left(\varphi_{k j}, \chi_{m i}\right)=2^{k / 2}\left(U^{k} \varphi, \chi_{m i}\right)=2^{k / 2} \sum_{\nu=0}^{\infty} c_{\nu}\left(r_{k+\nu}, \chi_{m i}\right)=2^{-(m-k) / 2} c_{m-k}
$$

в последнем равенстве мы учли обозначение $r_{k}$ и ортонормированность системы Хаара. Установлено равенство $\left(2.5_{1}\right)$.

Пусть теперь $k=m$. Если $j \neq i$, то $\Delta_{j}^{(k)} \cap \Delta_{i}^{(m)}=\varnothing($ см. раздел 1$)$. Поэтому ввиду (1.1) поточечное произведение $\varphi_{k j}(x) \chi_{m i}(x)$ тождественно равно нулю. Тем более, $\left(\varphi_{k j}, \chi_{m i}\right)=0$.

Если же $j=i$, то снова ввиду (1.1)

$$
\begin{aligned}
\left(\varphi_{k j}, \chi_{m i}\right) & =\left(\varphi_{m i}, \chi_{m i}\right)=\int_{\Delta_{i}^{(m)}} 2^{m / 2} \varphi\left(2^{m} x-i\right) 2^{m / 2} \chi\left(2^{m} x-i\right) d x \\
& =\int_{0}^{1} \varphi(x) \chi(x) d x=(\varphi, \chi)=c_{0},
\end{aligned}
$$

в последнем равенстве мы учли посылку предложения. Таким образом, установлено равенство $\left(2.5_{2}\right)$.

Наконец, пусть $k>m$.

Если $\Delta_{j}^{(k)} \cap \Delta_{i}^{(m)}=\varnothing$, то $\left(\varphi_{j k}, \chi_{m i}\right)=0$ - так же, как и вьше в случае $k=m, j=i$.

Если же $\Delta_{j}^{(k)} \cap \Delta_{i}^{(m)} \neq \varnothing$, то $\Delta_{j}^{(k)} \subset \Delta_{i}^{(m)}$, причем в силу строгого неравенства $k>m$ последнее включение строгое. Но поскольку $\Delta_{j}^{(m)}=\Delta_{2 i}^{(m+1)} \cup \Delta_{2 i+1}^{(m+1)}$, то либо $\Delta_{j}^{(k)} \subset \Delta_{2 i}^{(m+1)}$, либо $\Delta_{j}^{(k)} \subset \Delta_{2 i+1}^{(m+1)}$. Функция $\varphi_{k j}$ обрашается в нуль вне $\Delta_{j}^{(k)}\left(\right.$ см. (1.1)); на каждом из полуинтервалов $\Delta_{2 i}^{(m+1)}$ и $\Delta_{2 i+1}^{(m+1)}$ функция $\chi_{m i}$ постоянна (см. (1.2)). Поэтому скалярное произведение $\left(\varphi_{k j}, \chi_{m i}\right)$ пропорционально интегралу от функции $\varphi_{k j}$. Последний, в свою очередь, пропорционален интегралу от функции $\varphi$ (см. (1.1)), который, как видно из посылки предложения, равен нулю. Итак, $\left(\varphi_{k j}, \chi_{m i}\right)=0$. Установлено равенство $\left(2.5_{3}\right)$. 


\section{2. Леммы.}

ЛЕмма 1. Пусть система $\left(\psi_{n}\right)$ сжатий и сдвигов функиии $\psi$ является базисом Рисса. Тогда системы сжстий и сдвигов функиий

$$
\varphi=\sum_{k=0}^{\infty} \sum_{j=0}^{2^{k}-1} c_{k j} \psi_{k j} \quad u \quad \omega=\sum_{k=0}^{\infty} \sum_{j=0}^{2^{k}-1} c_{k j} \chi_{k j}
$$

одновременно являются или не являются базисами Рисса.

ДокАзАтельство. Так как $\left(\psi_{n}\right)$ - базис Рисса, то ввиду отмеченного в разделе 1 необходимого условия базисности функция $\psi$ имеет нулевой интеграл, т.е. $\psi \in L_{2}^{0}$; тогда $\psi_{n} \in L_{2}^{0}$ для всех $n \in \mathbb{N}$. Значит, система $\left(\psi_{n}\right), n \in \mathbb{N}$, является базисом Рисса в $L_{2}^{0}$, что означает существование обратимого оператора $A$, действующего в пространстве $L_{2}^{0}$, такого, что $A \chi_{n}=\psi_{n}, n \in \mathbb{N}$. В таком случае на основании предложения 2 будем иметь $A \omega_{n}=\varphi_{n}, n \in \mathbb{N}$, если установим равенство $A \omega=\varphi$.

Ho

$$
A \omega=A \sum_{k=0}^{\infty} \sum_{j=0}^{2^{k}-1} c_{k j} \chi_{k j}=\sum_{k=0}^{\infty} \sum_{j=0}^{2^{k}-1} c_{k j} A \chi_{k j}=\sum_{k=0}^{\infty} \sum_{j=0}^{2^{k}-1} c_{k j} \psi_{k j}=\varphi
$$

- здесь учли определения $\varphi$ и $\omega$ в формулировке леммы и равенство $A \chi_{k j}=\psi_{k j}$, равносильное доказанному равенству $A \chi_{n}=\psi_{n}$.

Итак, $A \omega_{n}=\varphi_{n}, n \in \mathbb{N}$. Кромеэтого, функции $\omega_{0}$ и $\varphi_{0}$ совпадают (каждая из них по определению равна $\chi[0,1))$. Следовательно, системы $\left(\omega_{n}\right)$ и $\left(\varphi_{n}\right)$ связаны обратимым ограниченньм преобразованием и если одна из них является базисом Рисса, то другая тоже.

Лемма 2. Если $A$ - обратимый оператор, перестановочный $c S_{n}, n \in \mathbb{N}$,

$$
A \chi=\sum_{k=0}^{\infty} c_{k} r_{k}
$$

mo

$$
A^{-1} \chi=\sum_{k=0}^{\infty} d_{k} r_{k}
$$

причем последовательности $\left(d_{k}\right) u\left(c_{k}\right)$ связаны соотношениями

$$
d_{0} c_{0}=1, \quad \sum_{k=0}^{m} d_{k} c_{m-k}=0, \quad m \in \mathbb{N} .
$$

ДОКАЗАТЕЛЬСТВО. Пусть

$$
A^{-1} \chi=\sum_{k=0}^{\infty} 2^{-k / 2} \sum_{j=0}^{2^{k}-1} d_{k j} \chi_{k j}
$$

- ряд Фурье-Хаара функции $A^{-1} \chi \in L_{2}^{0}$. 
Требуется доказать, что, во-первых, коэффициенты $d_{k j}=d_{k}$ не зависят от индекса $j$, что сразу повлечет за собой (2.6) (см. обозначение $r_{k}$ ); во-вторых, справедливы соотношения (2.7). Для этого воспользуемся индукцией по $k \in \mathbb{P}$.

Обозначим $\varphi=A \chi$. Используя перестановочность $A$ и $S_{n}, n \in \mathbb{N}$, получаем согласно предложению 2 равенство $\varphi_{n}=A \chi_{n}, n \in \mathbb{N}$. Записывая его в виде $\varphi_{k j}=A \chi_{k j}$ и учитьвая $(2.8)$, получаем

$$
\chi=A A^{-1} \chi=\sum_{k=0}^{\infty} 2^{-k / 2} \sum_{j=0}^{2^{k}-1} d_{k j} A \chi_{k j}=\sum_{k=0}^{\infty} 2^{-k / 2} \sum_{j=0}^{2^{k}-1} d_{k j} \varphi_{k j} .
$$

При $k=0$ имеем всего один коэффициент $d_{k j}$; его обозначим $d_{0}$. На основании полученного в (2.9) представления для $\chi$ и равенства $\left(2.5_{3}\right)$ с $m=0$ находим

$$
\begin{aligned}
1 & =(\chi, \chi)=\left(\sum_{k=0}^{\infty} 2^{-k / 2} \sum_{j=0}^{2^{k}-1} d_{k j} \varphi_{k j}, \chi\right) \\
& =d_{0}(\varphi, \chi)+\sum_{k=1}^{\infty} 2^{-k / 2} \sum_{j=0}^{2^{k}-1} d_{k j}\left(\varphi_{k j}, \chi\right)=d_{0} c_{0},
\end{aligned}
$$

в последнем равенстве мы учли также посылку леммы.

Выполним следуюший шаг индукции. Пусть $m \in \mathbb{N}$. Предположим, что для всех $0 \leqslant k \leqslant m-1,0 \leqslant j<2^{k}$ вьполняется равенство $d_{k j}=d_{k}$; докажем его для $k=m$.

Снова используя (2.9), а также равенства $(2.5)$, для $0 \leqslant i<2^{m}$ получаем

$$
\begin{aligned}
0= & \left(\chi, \chi_{m i}\right)=\left(\sum_{k=0}^{\infty} 2^{-k / 2} \sum_{j=0}^{2^{k}-1} d_{k j} \varphi_{k j}, \chi_{m i}\right) \\
= & \sum_{k=0}^{m-1} 2^{-k / 2} d_{k} \sum_{j=0}^{2^{k}-1}\left(\varphi_{k j, \chi_{m i}}\right)+2^{-m / 2} \sum_{j=0}^{2^{m}-1} d_{m j}\left(\varphi_{m j}, \chi_{m i}\right) \\
& +\sum_{k=m+1}^{\infty} 2^{-k / 2} \sum_{j=0}^{2^{k}-1} d_{k j}\left(\varphi_{k j}, \chi_{m i}\right) \\
= & 2^{-m / 2} \sum_{k=0}^{m-1} d_{k} c_{m-k}+2^{-m / 2} d_{m i} c_{0} .
\end{aligned}
$$

Поскольку последняя сумма нулевая, то, во-первых, видно, что $d_{m i}$ не зависит от $i$ $\left(c_{0} \neq 0\right.$ в силу уже доказанного равенства $\left.d_{0} c_{0}=1\right)$. Во-вторых, положив $d_{m i}=d_{m}$ и домножив нулевую сумму на $2^{m / 2}$, придем к соотношению $(2.7)$.

Лемма 3. Если оператор А перестановочен с $S_{n}, n \in \mathbb{N}$,

$$
A \chi=\sum_{k=0}^{\infty} c_{k} r_{k}
$$

то функиия

$$
u(z)=\sum_{k=0}^{\infty} c_{k} z^{k}
$$

принадлежит $H^{\infty}$. 
ДоКАЗАТЕЛЬСТВО. Обозначим через $R$ замкнутую линейную оболочку функции Радемахера. Пусть $H^{2}$ - пространство аналитических функций, последовательность коэффициентов ряда Тейлора (в нуле) которых суммируема с квадратом.

В силу равенства $(2.4)$ функции $U^{k} \chi$ совпадают с функциями Радемахера. Последние образуют ортонормированньй базис в $R$, поэтому по известной терминологии [3, c. 81$]$ сужение оператора $U$ на $R$ унитарно эквивалентно одностороннему сдвигу. Определив оператор $F$ из $R$ в $H^{2}$ равенством $F r_{k}=z^{k}$, видим, что оператор $F U E^{-1}$ есть тот самьй односторонний сдвиг (умножение на $z$ в $H^{2}$ ).

Покажем, что $R$ инвариантно относительно оператора $A$. Для этого достаточно показать, что $A r_{m} \in R$ для любого $m \in \mathbb{N}$.

Заметим, что оператор $A$ перестановочен с $U$, так как он перестановочен с $S(0)$ и $S(1)$, поскольку это частные случаи $S_{n}$. Тогда, учитьвая также равенство $(2.4)$ и условие леммы, получаем

$$
A r_{m}=A U^{m} \chi=U^{m} A \chi=U^{m} \sum_{k=0}^{\infty} c_{k} r_{k}=\sum_{k=0}^{\infty} c_{k} U^{m+k} \chi=\sum_{k=0}^{\infty} c_{k} r_{m+k}
$$

Таким образом, $A r_{m} \in R$. Инвариантность $R$ относительно $A$ установлена.

Последнее позволяет корректно определить оператор $B=F A F^{-1}$ в $H^{2}$. Так как $A$ перестановочен с $U$, то $B$ перестановочен с односторонним сдвигом $F U F^{-1}$. Следовательно $[3$, с. 80$]$, оператор $B$ есть оператор умножения на функцию из $H^{\infty}$. Покажем, что это функция $u(z)$, введенная в условии леммы.

Учитывая определения $B, F$ и представление $A r_{m}$ в последней цепочке равенств, получаем

$$
B z^{m}=F A F^{-1} z^{m}=F A r_{m}=F \sum_{k=0}^{\infty} c_{k} r_{m+k}=\sum_{k=0}^{\infty} c_{k} z^{m+k}=u(z) z^{m} .
$$

Лемма 4. Существует оператор $T$, действующий в пространстве $L_{2}^{0}$, изометрический, вполне неунитарный (т.е. не являющийся унитарным ни в одном ненулевом подпространстве пространства $L_{2}^{0}$ ) и такой, что

$$
T^{k} \chi_{n}=S_{n} r_{k}, \quad k \in \mathbb{P}, \quad n \in \mathbb{N} \text {. }
$$

ДокАЗАТЕЛЬСТво. Для $n \in \mathbb{N}$ положим

$$
T \chi_{n}=2^{-1 / 2}\left(\chi_{2 n}+\chi_{2 n+1}\right) .
$$

Продолжив оператор $T$ по линейности на конечные линейные комбинации функций $\chi_{n}$, будем иметь

$$
\left\|T \sum a_{n} \chi_{n}\right\|^{2}=\frac{1}{2}\left\|\sum a_{n} \chi_{2 n}+\sum a_{n} \chi_{2 n+1}\right\|^{2}=\sum\left|a_{n}\right|^{2},
$$

откуда вытекает возможность продолжения оператора $T$ до изометрического оператора во всем пространстве $L_{2}^{0}$. 
Для доказательства того факта, что оператор $T$ вполне неунитарньй, сначала установим для сопряженного оператора $T^{*}$ неравенство

$$
\left\|\left(T^{*}\right)^{k} f\right\|^{2} \leqslant \sum_{n=2^{k}}^{\infty}\left|\left(f, \chi_{n}\right)\right|^{2}, \quad f \in L_{2}^{0} .
$$

Заметим, что сопряженный оператор мы понимаем в смысле пространства $L_{2}^{0}$.

Докажем неравенство (2.12) индукцией по $k \in \mathbb{P}$. При $k=0$ имеем

$$
\left\|\left(T^{*}\right)^{k} f\right\|^{2}=\|f\|^{2}=\sum_{n=1}^{\infty}\left|\left(f, \chi_{n}\right)\right|^{2} .
$$

Предположим, что неравенство (2.12) справедливо при некотором $k$, и получим его при $k+1$.

Используя (2.12) с $g=T^{*} f$ вместо $f$ и учитьвая определение (2.11) оператора $T$, находим

$$
\begin{aligned}
\left\|\left(T^{*}\right)^{k+1} f\right\|^{2} & =\left\|\left(T^{*}\right)^{k} g\right\|^{2} \leqslant \sum_{n=2^{k}}^{\infty}\left|\left(g, \chi_{n}\right)\right|^{2} \\
& =\sum_{n=2^{k}}^{\infty}\left|\left(f, T \chi_{n}\right)\right|^{2}=\frac{1}{2} \sum_{n=2^{k}}^{\infty}\left|\left(f, \chi_{2 n}\right)+\left(f, \chi_{2 n+1}\right)\right|^{2} \\
& \leqslant \sum_{n=2^{k}}^{\infty}\left(\left|\left(f, \chi_{2 n}\right)\right|^{2}+\left|\left(f, \chi_{2 n+1}\right)\right|^{2}\right)=\sum_{n=2^{k+1}}^{\infty}\left|\left(f, \chi_{n}\right)\right|^{2} .
\end{aligned}
$$

Неравенство (2.12) при $k+1$ установлено.

Из неравенства (2.12) следует, что $\left(T^{*}\right)^{k} f \rightarrow 0$ при $k \rightarrow \infty$ для любой функции $f \in L_{2}^{0}$. Отсюда получаем вполне неунитарность оператора $T$ стандартным образом.

Предположим, что оператор $T$ унитарный в подпространстве $\mathscr{L} \subset L_{2}^{0}$. Тогда для любой функции $f \in \mathscr{L}$ и любого $k$ будет вьполняться равенство $\|f\|=\left\|\left(T^{*}\right)^{k} f\right\|$. Устремляя здесь $k \rightarrow \infty$, получим $f=0$, откуда $\mathscr{L}=\{0\}$, что и требовалось.

Наконец, докажем равенство (2.10); сделаем это индукцией по $k$.

При $k=0$ имеем

$$
T^{k} \chi_{n}=\chi_{n}=S_{n} \chi=S_{n} r_{0}=S_{n} r_{k} ;
$$

мы учли определение (2.1) оператора $S_{n}$ и равенство $r_{0}=\chi$.

Предположим по индукции, что равенство (2.10) справедливо при некотором $k$ для любого $n \in \mathbb{N}$, и получим его при $k+1$.

Учитывая определение (2.11) оператора $T$ и равенство $(2.10)$ с $\chi_{2 n}$ и $\chi_{2 n+1}$ вместо $\chi_{n}$, находим

$$
T^{k+1} \chi_{n}=T^{k} T \chi_{n}=T^{k} 2^{-1 / 2}\left(\chi_{2 n}+\chi_{2 n+1}\right)=2^{-1 / 2}\left(S_{2 n}+S_{2 n+1}\right) r_{k} .
$$

Пусть $n=2^{k}+\sum_{\nu=1}^{k} \varepsilon_{\nu} 2^{k-\nu}-$ двоичное разложение и $\varepsilon \in\{0,1\}$. Тогда $2 n+\varepsilon=$ $2^{k+1}+\sum_{\nu=1}^{k+1} \varepsilon_{\nu} 2^{k+1-\nu}$, если положить $\varepsilon_{k+1}=\varepsilon$. Отсюда и в силу представления $(2.2)$ получаем

$$
S_{2 n+\varepsilon}=S_{n} S(\varepsilon) \text {. }
$$

Подставим это равенство в правую часть последней цепочки равенств, учтем определение оператора $U$ (см. предложение 3 ), равенство (2.4). Найдем

$$
T^{k+1} \chi_{n}=S_{n} 2^{-1 / 2}(S(0)+S(1)) r_{k}=S_{n} U r_{k}=S_{n} U^{k+1} \chi=S_{n} r_{k+1} .
$$

Равенство (2.10) установлено. 
ЛЕмма 5. Пусть функиия $\varphi$ разлагается в ряд

$$
\varphi=\chi+\sum_{k=1}^{\infty} \sum_{j=0}^{2^{k}-1} c_{k j} \chi_{k j}
$$

Для $k \in \mathbb{N}$ положимм

$$
\theta_{k}=\left(\sum_{j=0}^{2^{k}-1}\left|c_{k j}\right|^{2}\right)^{1 / 2}
$$

и предположим, что сходится ряд

$$
\theta=\sum_{k=1}^{\infty} \theta_{k}
$$

Тогда для любой конечной последовательности комплексных чисел $\left\{a_{n}\right\}$ выполняется неравенство

$$
\left\|\sum a_{n}\left(\varphi_{n}-\chi_{n}\right)\right\| \leqslant \theta\left(\sum\left|a_{n}\right|^{2}\right)^{1 / 2}
$$

ДокАЗАТЕЛЬСТВо. Для функции $f \in L_{2}^{0}$ положим

$$
\sigma_{N}(f)=\sum_{k=0}^{N} \sum_{j=0}^{2^{k}-1}\left(f, \chi_{k j}\right) \chi_{k j}
$$

- это частная сумма Фурье-Хаара функции $f$.

Обозначим $\psi=\varphi-\chi$. Учитывая определение $(2.1)$ оператора $S_{n}$, имеем

$$
\sigma_{N}\left(\sum a_{n}\left(\varphi_{n}-\chi_{n}\right)\right)=\sum a_{n} \sigma_{n} S_{n} \psi
$$

Подставляя сюда разложение

$$
\psi=\sum_{k=1}^{\infty} \sum_{j=0}^{2^{k}-1} c_{k j} \chi_{k j}=\sum c_{m} \chi_{m}=\sum c_{m} S_{m} \chi
$$

находим

$$
\sigma_{N}\left(\sum a_{n}\left(\varphi_{n}-\chi_{n}\right)\right)=\sum \sum a_{n} c_{m} \sigma_{N} S_{n} S_{m} \chi
$$

Из представления (2.2) следует, что $S_{n} S_{m}=S_{p}$ для некоторого $p$, причем $k(p)=$ $k(n)+k(m)$.

Отсюда и из очевидных соотношений

$$
\sigma_{N} S_{p} \chi= \begin{cases}S_{p} \chi, & k(p) \leqslant N \\ 0, & k(p)>N\end{cases}
$$


а также из равенства (2.14) получаем

$$
\sigma_{N}\left(\sum a_{n}\left(\varphi_{n}-\chi_{n}\right)\right)=\sum_{k(n)+k(m) \leqslant N} a_{n} c_{m} S_{n} S_{m} \chi=\sum_{k=1}^{N} \sum_{k(m)=k} c_{m} \sum_{k(n)=0}^{N-k} a_{n} S_{n} S_{m} \chi,
$$

в первой сумме последнего выражения суммирование начинается с $k=1$, а не с $k=0$, ввиду того, что $c_{m}=0$ при $k=k(m)=0$ (см. разложение $\left.(2.13)\right)$.

Зафиксируем $k$ и покажем, что семейство $\left(S_{n} S_{m} \chi\right), n \in \mathbb{N}, k(m)=k$, ортонормировано.

Рассмотрим упорядоченную пару $(n, m)$, где $k(m)=k$, а в остальном $n, m \in \mathbb{N}$ произвольные.

Пусть

$$
n=2^{k(n)}+\sum_{\nu=1}^{k(n)} \varepsilon_{\nu} 2^{k(n)-\nu}, \quad m=2^{k}+\sum_{\nu=1}^{k} \zeta_{\nu} 2^{k-\nu}
$$

- двоичные разложения.

Положим

$$
p=2^{k+k(n)}+2^{k} \sum_{\nu=1}^{k(n)} \varepsilon_{\nu} 2^{k(n)-\nu}+\sum_{\nu=1}^{k} \zeta_{\nu} 2^{k-\nu}
$$

Тогда

$$
S_{n} S_{m}=\prod_{\nu=1}^{k(n)} S\left(\varepsilon_{\nu}\right) \prod_{\nu=1}^{k} S\left(\zeta_{\nu}\right)=S_{p}
$$

причем $k(p)=k+k(n) \geqslant k$ (см. представление $(2.2))$.

Обратно, возьмем $p \in \mathbb{N}, k(p) \geqslant k$ и обозначим $k(n)=k(p)-k$. Нетрудно видеть, что двоичному разложению числа $p$ можно придать вид (2.17). Тогда, определив числа $n$ и $m$ равенствами (2.16), снова получим (2.18).

Таким образом, между упорядоченньми парами $(n, m), n \in \mathbb{N}, k(m)=k$, и числами $p, k(p) \geqslant k$, построено с помощью разложений (2.16) и (2.17) взаимнооднозначное соответствие, которое равенством (2.18) переносится на операторы $S_{p}$.

Следовательно, семейства $\left(S_{n} S_{m} \chi\right), n \in \mathbb{N}, k(m)=k$, и $\left(S_{p} \chi\right), k(p) \geqslant k$, одновременно ортонормированы или нет.

Ортонормированность же второго семейства следует из определения (2.1) операторов $S_{p}$ и ортонормированности системы Хаара.

Итак, установлена ортонормированность семейства $\left(S_{n}, S_{m} \chi\right), n \in \mathbb{N}, k(m)=k$, для любого $k$.

Отсюда и из равенства (2.15) находим

$$
\begin{aligned}
& \left\|\sigma_{n}\left(\sum a_{n}\left(\varphi_{n}-\chi_{n}\right)\right)\right\| \leqslant \sum_{k=1}^{N}\left\|\sum_{k(m)=k} c_{m} \sum_{k(n)=0}^{N-k} a_{n} S_{n} S_{m} \chi\right\| \\
& \quad=\sum_{k=1}^{N}\left(\sum_{k(m)=k}\left|c_{m}\right|^{2} \sum_{k(n)=0}^{N-k}\left|a_{n}\right|^{2}\right)^{1 / 2} \leqslant \sum_{k=1}^{N}\left(\sum_{k(m)=k}\left|c_{m}\right|^{2} \sum\left|a_{n}\right|^{2}\right)^{1 / 2} \\
& =\sum_{k=1}^{N}\left(\sum_{j=0}^{2^{k}-1}\left|c_{k j}\right|^{2}\right)^{1 / 2}\left(\sum\left|a_{n}\right|^{2}\right)^{1 / 2}=\sum_{k=1}^{N} \theta_{k}\left(\sum\left|a_{n}\right|^{2}\right)^{1 / 2} .
\end{aligned}
$$


Устремляя $N \rightarrow \infty$ и учитьвая, что $\left\|\sigma_{N}(f)\right\| \rightarrow\|f\|$, получаем доказьваемое неравенство.

\section{3. Доказательство теорем}

ДоКАЗАТЕЛЬСТВО ТЕОРЕМЫ 1. Ввиду леммы 1 теорему достаточно будет доказать для случая $\psi=\chi$. В этом случае разложение (1.3) из посылки теоремы примет вид

$$
\varphi=\sum_{k=0}^{\infty} 2^{-k / 2} c_{k} \sum_{j=0}^{2^{k}-1} \chi_{k j}=\sum_{k=0}^{\infty} c_{k} r_{k}
$$

(см. обозначения раздела 2).

Необходимость. Пусть система сжатий и сдвигов $\left(\varphi_{n}\right)$ является базисом Рисса. Докажем, что функция $u(z)$ из формулировки теоремы принадлежит групе $G\left(H^{\infty}\right)$.

Так же, как и в начале доказательства леммы 1 , убеждаемся в существовании обратимого оператора $A$, действующего в пространстве $L_{2}^{0}$ и такого, что $A \chi_{n}=\varphi_{n}, n \in \mathbb{N}$. Такой оператор в силу предложения 2 перестановочен с $S_{n}$, а ввиду (3.1) для него выполняется равенство

$$
A \chi=\varphi=\sum_{k=0}^{\infty} c_{k} r_{k}
$$

Согласно лемме 2

$$
A^{-1} \chi=\sum_{k=0}^{\infty} d_{k} r_{k}
$$

причем последовательности $\left(d_{k}\right)$ и $\left(c_{k}\right)$ связаны соотношениями

$$
d_{0} c_{0}=1, \quad \sum_{k=0}^{m} d_{k} c_{m-k}=0, \quad m \in \mathbb{N} .
$$

Применяя лемму 3 дважды: к оператору $A$ и последовательности $\left(c_{k}\right)$, затем к оператору $A^{-1}$ и последовательности $\left(d_{k}\right)$, находим, что функции

$$
u(z)=\sum_{k=0}^{\infty} c_{k} z^{k}, \quad v(z)=\sum_{k=0}^{\infty} d_{k} z^{k}
$$

принадлежат $H^{\infty}$. Применение леммы $3 \mathrm{k} A^{-1}$ законно в силу перестановочности $A^{-1}$ c $S_{n}$, вытекающей из перестановочности $A$ и $S_{n}$.

Тогда ввиду соотношений (3.2) получаем $u(z) v(z) \equiv 1$. Таким образом, $u(z) \in$ $G\left(H^{\infty}\right)$. Необходимость доказана.

Достаточность. Пусть функция $u(z)$ принадлежит $G\left(H^{\infty}\right)$. Требуется доказать, что система сжатий и сдвигов $\left(\varphi_{n}\right)$ является базисом Рисса.

Так как $u(z) \in G\left(H^{\infty}\right)$, то $1 / u=v \in H^{\infty}$. Пусть $T$ - оператор из леммы 4 . Для него существуют сильные операторные пределы

$$
u(T)=\lim _{t \rightarrow 1-0} \sum_{k=0}^{\infty} c_{k}(t T)^{k} \quad \text { для } \quad u(z)=\sum_{k=0}^{\infty} c_{k} z^{k}
$$


И

$$
v(T)=\lim _{t \rightarrow 1-0} \sum_{k=0}^{\infty} d_{k}(t T)^{k} \quad \text { для } \quad v(z)=\sum_{k=0}^{\infty} d_{k} z^{k},
$$

причем операторы $u(T)$ и $v(T)$ взаимно обратны, т.е.

$$
u(T) v(T)=I=v(T) u(T),
$$

где $I$ - тождественньй оператор в пространстве $L_{2}^{0}[4$, с. 131].

Используем свойство (2.10) оператора $T$ и определение (2.1) оператора $S_{n}, n \in \mathbb{N}$. Получаем

$$
\begin{aligned}
u(T) \chi_{n} & =\lim _{t \rightarrow 1-0} \sum_{k=0}^{\infty} c_{k}(t T)^{k} \chi_{n}=\lim _{t \rightarrow 1-0} \sum_{k=0}^{\infty} c_{k} t^{k} S_{n} r_{k} \\
& =S_{n} \lim _{t \rightarrow 1-0} \sum_{k=0}^{\infty} c_{k} t^{k} r_{k}=S_{n} \sum_{k=0}^{\infty} c_{k} r_{k}=S_{n} \varphi=\varphi_{n} .
\end{aligned}
$$

Доказанное равенство $u(T) \chi_{n}=\varphi_{n}$ означает, что система $\left(\varphi_{n}\right), n \in \mathbb{N}$, является базисом Рисса в $L_{2}^{0}$. Добавив к системе функцию $\varphi_{0}=\chi[0,1)$, получим базис Рисса в $L_{2}[0,1]$.

ДоКАЗАТЕЛЬСТво ТЕОРЕМЫ 2. Необходимость. Пусть для любой функции $\varphi \in$ $A\left(\psi,\left(b_{k}\right)\right)$ система ее сжатий и сдвигов является базисом Рисса. Требуется доказать, что

$$
\sum_{k=1}^{\infty} b_{k}<1
$$

Рассмотрим функцию

$$
\varphi=\psi-\sum_{k=1}^{\infty} 2^{-k / 2} b_{k} \sum_{j=0}^{2^{k}-1} \psi_{k j}
$$

Согласно определению 2 функция $\varphi$ принадлежит классу $A\left(\psi,\left(b_{k}\right)\right)$. Значит, система $\left(\varphi_{n}\right)$ является базисом Рисса. В силу доказанной теоремы 1 функция

$$
u(z)=1-\sum_{k=1}^{\infty} b_{k} z^{k}
$$

принадлежит $G\left(H^{\infty}\right)$ и, следовательно, удовлетворяет условию

$$
0<\delta=\inf |u(z)|, \quad|z|<1 \text {. }
$$

Отсюда ввиду неотрищательности коэффициентов $b_{k}$ и равенства $u(0)=1$ для $t \in(0,1)$ получаем неравенство $u(t) \geqslant \delta$, за ним и следующие неравенства:

$$
t^{n} \sum_{k=1}^{n} b_{k} \leqslant \sum_{k=1}^{n} b_{k} t^{k} \leqslant \sum_{k=1}^{\infty} b_{k} t^{k}=1-u(t) \leqslant 1-\delta .
$$

Устремив здесь сначала $t \rightarrow 1$, затем $n \rightarrow \infty$, окончательно получим неравенство (3.3). Необходимость доказана. 
Достаточность. Пусть выполнено условие (3.3). Требуется доказать, что для всякой функции $\varphi \in A\left(\psi,\left(b_{k}\right)\right)$ система $\left(\varphi_{n}\right)$ является базисом Рисса.

Пусть сначала $\psi=\chi$. Тогда функция $\varphi$ принадлежит классу $A\left(\chi,\left(b_{k}\right)\right)$ и разлагается в ряд

$$
\varphi=c_{0} \chi+\sum_{k=1}^{\infty} \sum_{j=0}^{2^{k}-1} c_{k j} \chi_{k j}
$$

коэффициенты которого удовлетворяют соотношениям (1.5). Согласно примечанию 4 к теореме 2 считаем $c_{0}=1$. Тогда в обозначениях леммы 5 на основании соотношений (5.1) и в силу неравенства (3.3) будем иметь

$$
\theta=\sum_{k=1}^{\infty} \theta_{k}=\sum_{k=1}^{\infty}\left(\sum_{j=0}^{2^{k}-1}\left|c_{k j}\right|^{2}\right)^{1 / 2} \leqslant \sum_{k=1}^{\infty} b_{k}<1
$$

Применив лемму 5, получим неравенство

$$
\left\|\sum a_{n}\left(\varphi_{n}-\chi_{n}\right)\right\| \leqslant \theta\left(\sum\left|a_{n}\right|^{2}\right)^{1 / 2}
$$

для любой конечной последовательности комплексных чисел $\left\{a_{n}\right\}$.

Так как в полученном неравенстве $\theta<1$, то по теореме Пэли-Винера (см. например, $\left[5\right.$, с. 225]) система $\left(\varphi_{n}\right)$ будет базисом, для которого при любой функции $f \in L_{2}[0,1]$ выполняются неравенства

$$
\left.(1-\theta)\|f\| \leqslant\left.\left(\sum \mid f, \varphi_{n}\right)\right|^{2}\right)^{1 / 2} \leqslant(1+\theta)\|f\| .
$$

Подобные неравенства характеризуют базисы Рисса [6, с. 69].

Достаточность для случая $\psi=\chi$ доказана. Произвольный случай следует из леммы 1 и определения 2.

Автор искренне благодарен В. И. Филиппову за постановку задачи, полезные обсуждения и постоянное внимание в процессе работы.

\section{СПИСОК ЦИТИРОВАННОЙ ЛИТЕРАТУРЫ}

[1] Filippov V. I., Oswald P. Representation in $L_{p}$ by series of translates and dilates of one function // J. Approx. Theory. 1995. V. 82. № 1. P. 15-29.

[2] Сабурова Т.Н. О базисах в $C[0,1]$ типа Фабера-Шаудера // Теория функций и приближений. Ч. 3. Тр. 3-й Саратовской зимней школы (Саратов, 1986). Саратов, 1988. С. 44-46.

[3] Халмош П. Гильбертово пространство в задачах. М.: Мир, 1970.

[4] Секефальви-Надь Б., Фояш Ч. Гармонический анализ операторов в гильбертовом пространстве. М.: Мир, 1970.

[5] Рисс Ф., Секефальви-Надь Б. Лекции по функциональному анализу. М.: Мир, 1979.

[6] Крейн С.Г. (ред. ) Функциональный анализ. Серия "Справочная математическая библиотека". M.: Наука, 1972. 